\title{
Return to Play After Arthroscopic Stabilization for Posterior Shoulder Instability-A Systematic Review
}

\author{
Jordan W. Fried, B.M., Eoghan T. Hurley, M.B., B.Ch., M.Ch., Matthew L. Duenes, B.S., \\ Amit K. Manjunath, B.S., Mandeep Virk, M.D., Guillem Gonzalez-Lomas, M.D., and \\ Kirk A. Campbell, M.D.
}

\begin{abstract}
Purpose: To ascertain the rate and timing of return to play (RTP) and the availability of specific criteria for safe RTP after arthroscopic posterior shoulder stabilization. Methods: Medline, EMBASE, and the Cochrane Library were searched according to the Preferred Reporting Items for Systematic Reviews and Meta-Analyses guidelines to find studies on arthroscopic posterior shoulder stabilization. Studies were included if they reported RTP data or rehabilitation protocols and excluded if concomitant procedures influenced the rehabilitation protocol. Rate and timing of RTP, along with rehabilitation protocols, were assessed. Results: This review found 25 studies, including 895 cases, meeting the study's inclusion criteria. The majority of patients were male $(82.7 \%)$, with an age range of 14 to 66 years and a follow-up range of 4 to 148.8 months. The overall RTP rate ranged from $62.7 \%$ to $100.0 \%$, and $50.0 \%$ to $100.0 \%$ returned to the same level of play. Among collision athletes, the overall rate of RTP was $80.0 \%$ to $100.0 \%$, with $69.2 \%-100.0 \%$ returning to the same level of play. In overhead athletes, the overall rate of RTP was $85.2 \%$ to $100.0 \%$, with $55.6 \%$ to $100.0 \%$ returning to the same level of play. Four studies (128 patients) specifically addressed the timing of RTP, and the range to RTP was 4.3 to 8.6 months. Specific RTP criteria were reported in a majority of studies $(60 \%)$, with the most reported item being restoration of strength $(44 \%)$. Conclusion: There is a high rate of return to sport after arthroscopic posterior shoulder stabilization, ranging from 4.3 to 8.6 months after surgery. Return to preinjury level is higher for collision athletes compared with overhead athletes. However, there is inadequate reporting of RTP criteria in the current literature, with no clear timeline for when it is safe to return to sport. Level of Evidence: IV, systematic review of level II to IV studies
\end{abstract}

$\mathbf{P}$ osterior shoulder instability (PSI) represents an estimated $2 \%$ to $10 \%$ of all shoulder instability cases. $^{1-5}$ PSI often presents insidiously, as the most common symptom is shoulder pain and not an acute instability event. Repetitive microtrauma to the shoulder, atraumatic causes including baseline ligamentous laxity, and acute traumatic events have been proposed and suspected in PSI. ${ }^{4,6-8}$ PSI is commonly seen in athletes who perform high-demand, dynamic

From the Department of Orthopaedic Surgery, Division of Sports Medicine, NYU Langone Health, New York, New York, U.S.A.

The authors report that they have no conflicts of interest in the authorship and publication of this article. Full ICMJE author disclosure forms are available for this article online, as supplementary material.

Received February 18, 2020; accepted August 19, 2020.

Address correspondence to Jordan W. Fried, Division of Sports Medicine, NYU Langone Orthopedic Center, 333 East 38th Street, New York, NY 10016, U.S.A.E-mail: jordanfried117@gmail.com

(C) 2020 THE AUTHORS. Published by Elsevier Inc. on behalf of the Arthroscopy Association of North America. This is an open access article under the CC BY-NC-ND license (http://creativecommons.org/licenses/by-nc-nd/4.0/).

2666-061X/20197

https://doi.org/10.1016/j.asmr.2020.08.007 posterior-loading activities, such as weightlifters and American football linemen, as well as athletes in sports that reward increased shoulder range of motion, including swimming and gymnastics. It is occasionally seen in the young military population. ${ }^{4,5,8-12}$ PSI may be treated conservatively or operatively, with management dictated by patient factors (age, compliance, comorbidities), evidence of any bony pathology involving the glenohumeral joint, and mechanism of instability. ${ }^{2,13}$

Burkhead and Rockwood ${ }^{14}$ found nonoperative management to be less successful in patients with a history of a traumatic event, as $16 \%$ of patients with a traumatic event had clinical success as opposed to $70 \%$ to $89 \%$ of patients without a traumatic event. Surgery is commonly considered when patients continue to experience instability symptoms and fail nonoperative treatment. ${ }^{7,14,15}$ However, although surgical correction for PSI has provided improved clinical outcomes, the rates of return to play (RTP) for PSI are unclear. ${ }^{9,10,16-20}$ Additionally, there is no consensus for guidelines or criteria on how to allow patients to return to play safely after posterior shoulder stabilization. 


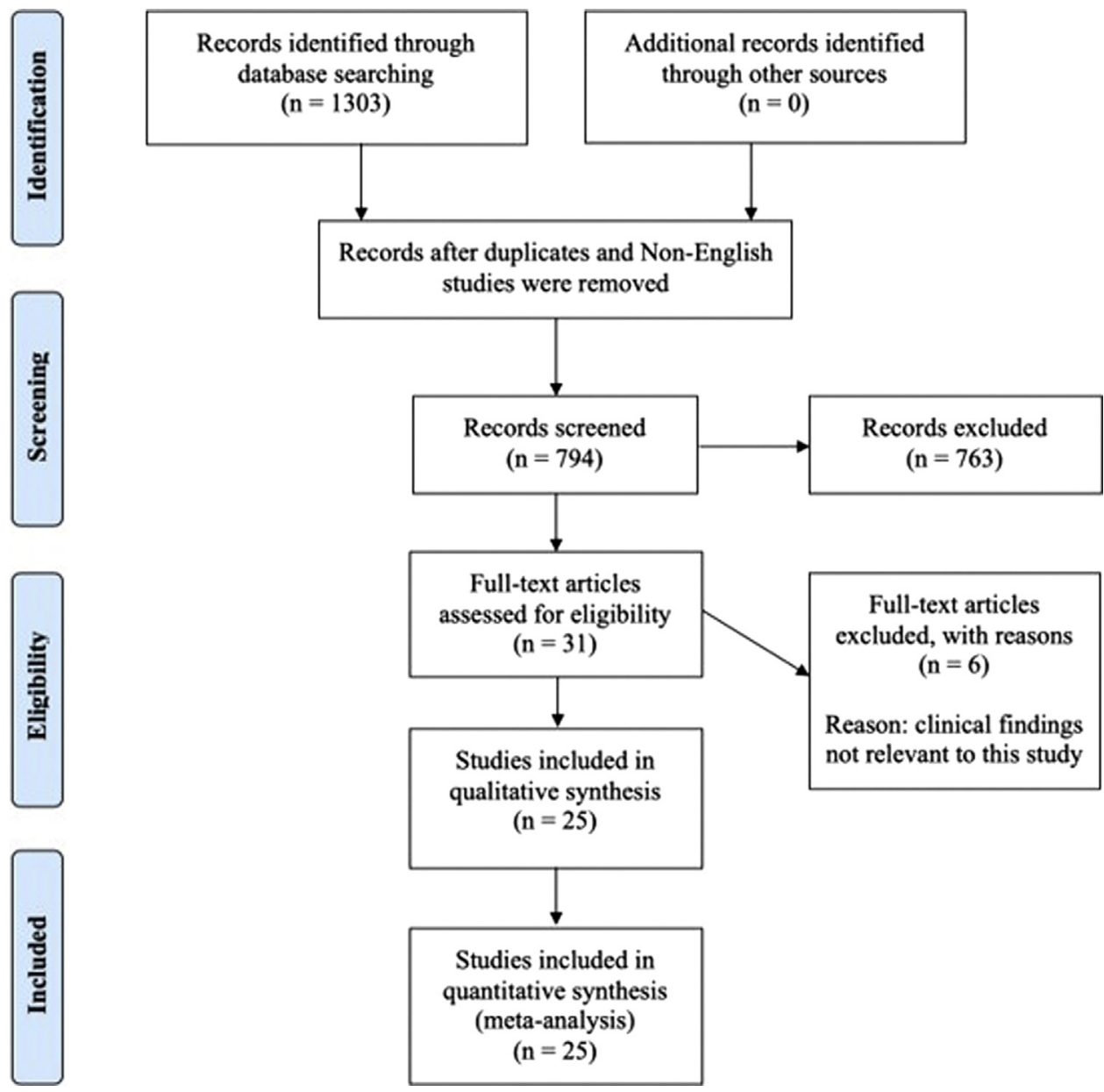

Fig 1. Preferred Reporting Items for Systematic Reviews and MetaAnalyses (PRISMA) flow chart.

The purpose of this systematic review is to ascertain the rate and timing of RTP and the availability of specific criteria for safe RTP after arthroscopic posterior shoulder stabilization. The hypothesis is that there is a significant rate of RTP after arthroscopic posterior stabilization but substantial differences in RTP protocols.

\section{Methods}

\section{Search Strategy and Study Selection}

To collate the existing evidence related to rehabilitation protocol and RTP after posterior shoulder stabilization, a systematic review was performed according to the Preferred Reporting Items for Systematic Reviews and Meta-Analyses (PRISMA) guidelines. Eligibility criteria for this review consisted of clinical studies of patients undergoing arthroscopic posterior shoulder stabilization in which rehabilitation protocols or RTP data were reported. Further study characteristics required for eligibility included publication in a peerreviewed journal and availability of the full text of the study. Studies were deemed ineligible for this review if they included concomitant procedures that explicitly influenced the rehabilitation protocol or patients with multidirectional laxity. Review articles, case reports, surgical technique articles, biomechanical studies, and non-English language articles were not included.

Screening, eligibility, and inclusion were determined by 2 independent reviewers (J.W.F. and E.T.H.). The Medline, EMBASE, and Cochrane Library databases were searched from their inception to December 20, 2019, using the following algorithm: posterior AND shoulder instability AND (arthrosc*). The titles and abstracts of returned results were screened according to the described eligibility criteria (Fig 1). Potentially relevant studies were identified, and the associated full text was reviewed. The reference lists of all relevant studies were screened for any articles not identified through the database search. Studies were included based on agreement of both independent reviewers. Any instances of disagreement were resolved through consultation with the senior author.

\section{Assessment of Level and Quality of Evidence}

The level of evidence (LOE) of the included studies was evaluated based on the criteria from the Oxford Centre for Evidence-Based Medicine. ${ }^{21}$ The quality of 
Table 1. Study Characteristics

\begin{tabular}{|c|c|c|c|c|c|c|}
\hline Reference & $\mathrm{LOE}$ & QOE & Patients & Age $(y)$ & Male sex $(\%)$ & Follow-up (mo) \\
\hline Andrieu et al. $2017^{25}$ & III & 63 & 101 & 28.8 & 74.2 & 49.7 \\
\hline Arner et al. $2015^{26}$ & IV & 79 & 56 & $17.9(14.8$ to 25.5$)$ & 100 & 44.7 (24 to 98$)$ \\
\hline Bahk et al. $2010^{27}$ & IV & 62 & 29 & $26.3(18.3$ to 43.4$)$ & 97 & $66(24$ to 148.8$)$ \\
\hline Badge et al. $2009^{12}$ & IV & 87 & 11 & $24.8(15$ to 36$)$ & 100 & $32(17$ to 54$)$ \\
\hline Bradley et al. $2006^{18}$ & II & 92 & 100 & $23.3(15$ to 61$)$ & 77 & 27.7 ( 12 to 77$)$ \\
\hline Bradley et al. $2013^{28}$ & II & 72 & 200 & $24.3(15$ to 65$)$ & 79 & $36.7(12$ to 115$)$ \\
\hline Castagna et al. $2007^{29}$ & IV & 63 & 9 & 25.2 & 77.8 & $34.2(28$ to 39$)$ \\
\hline Eckenrode et al. $2009^{30}$ & IV & 65 & 5 & $20.2(18$ to 22$)$ & 100 & NR \\
\hline Garret et al. $2017^{31}$ & III & 49 & 25 & $30(16$ to 45$)$ & 64 & NR \\
\hline Hines et al. $2018^{37}$ & III & 52 & 32 & $30.8(20$ to 47$)$ & 93.8 & 53.7 (25 to 82$)$ \\
\hline Katthagen et al. $2017^{33}$ & III & 69 & 38 & $27.6(13$ to 66$)$ & 92.1 & $49.2(24$ to 93.6$)$ \\
\hline Kercher et al. $2019^{35}$ & IV & 76 & 32 & $20.5(16$ to 41$)$ & 100 & 41.58 (24 to 92$)$ \\
\hline Kim et al. $2003^{36}$ & IV & 72 & 15 & $21(17$ to 25$)$ & 93 & $39(31$ to 47$)$ \\
\hline Kraeutler et al. $2018^{34}$ & III & 74 & 22 & $26.6(17$ to 45$)$ & 95 & $43.2(26.4$ to 88.8$)$ \\
\hline Lacheta et al. $2019^{32}$ & IV & 72 & 7 & $23.5(17$ to 43$)$ & 100 & $96(36$ to 120$)$ \\
\hline Lenart et al. $2012^{19}$ & IV & 70 & 19 & $21.4(15$ to 33$)$ & 81 & $36(12$ to 67$)$ \\
\hline Mair et al. $1998^{9}$ & IV & 73 & 9 & $18.8(16$ to 21$)$ & 100 & $30(24$ to 42$)$ \\
\hline McClincy et al. $2015^{38}$ & III & 77 & 48 & 17.8 & 71 & 37 (12 to 97$)$ \\
\hline McClincy et al. $2020^{39}$ & IV & 80 & 68 & $17.2(14$ to 19$)$ & NR & 45 \\
\hline Papendick and Savoie $1995^{20}$ & IV & 62 & 41 & $23(15$ to 42$)$ & NR & $10(4$ to 41$)$ \\
\hline Radkowski et al. $2008^{40}$ & II & 90 & 98 & 22.9 & 76.5 & 27 \\
\hline Robins et al. $2017^{41}$ & IV & 58 & 42 & NR & NR & 39.6 \\
\hline Wanich et al. $2012^{42}$ & IV & 68 & 12 & $20.3(16$ to 33$)$ & 100 & 33.6 (18 to 64$)$ \\
\hline Wolf and Eakin $1998^{43}$ & IV & 60 & 5 & $26(14$ to 54$)$ & 79 & $33(24$ to 45$)$ \\
\hline Wooten et al. $2015^{44}$ & IV & 74 & 22 & 17.3 & 86.30 & $63(24$ to 115$)$ \\
\hline
\end{tabular}

Data for age and follow-up are mean (range).

LOE, level of evidence; NR, not reported; QOE, quality of evidence.

studies was assessed based on the criteria from the Modified Coleman Methodology Score as performed by Ramponi et al. ${ }^{22}$ The methodological quality of the RTP guidelines described in each study was assessed based on the criteria outlined by Zaman et al. ${ }^{23}$ Instances of scoring discrepancy were resolved through consultation with the senior author to reach a consensus.

\section{Data Extraction}

Two reviewers independently extracted data in duplicate from the included studies using a predesigned data collection form. Screening was performed to remove duplicate patients between studies. Demographic variables included total number of patients, sex ratio, patient age, and follow-up time. Variables related to RTP were recorded, including percentage of patients returning to play, ability to return to the preoperative level of play, patient-reported timing of return to athletic activity, and reasons for not returning to sport.

\section{Statistical Analysis}

Statistical analysis was performed using SPSS (release 2013; IBM SPSS Statistics for Macintosh, version 22.0. Armonk, NY). Descriptive statistics were determined for all categorical and continuous variables. Categorical variables were reported as frequencies with percentages, and continuous variables were reported as a weighted mean with an estimated standard deviation.
The quality of RTP for each study was determined according to the quality of the RTP guidelines outlined by Zaman et al. ${ }^{23}$ For all analyses, $P$ values $<.05$ were considered to be statistically significant.

\section{Results}

Initially, 1303 studies were identified. After removal of duplicates and non-English studies, 794 studies were further analyzed. After application of inclusion and exclusion criteria, 25 studies reporting on 996 shoulders were included in the final analysis (Fig 1).

\section{Study Characteristics}

Overall, 25 clinical studies (LOE II, 3; LOE III, 6; LOE IV, 16) reported RTP rates, including 996 shoulders. There were 740 males and 155 females, with ages ranging from 14.8 to 66 years, who were followed up for 4 to 148.8 months (Table 1).

\section{Rate and Time of RTP}

Rates of return to play were reported in 25 studies. The overall rate of RTP was $62.7 \%$ to $100 \%$; in 18 studies, $50.0 \%$ to $100 \%$ returned to the same level of play. Four studies (128 patients) reported time of return to play, which was 4.3 to 8.6 months (Table 2).

\section{Rate of RTP Among Collision and Overhead Sports}

Among collision athletes, the overall rate of return to play was $80 \%$ to $100 \%$, with $69.2 \%$ to $100 \%$ returning 
Table 2. Rate and Time of Return to Play

\begin{tabular}{|c|c|c|c|}
\hline Reference & Return to Play (\%) & $\begin{array}{c}\text { Return to Play at Same or } \\
\text { Higher Level }(\%)\end{array}$ & Time $(\mathrm{mo})$ \\
\hline Andrieu et al. $2017^{25}$ & 62.7 & NR & 7.7 \\
\hline Arner et al. $2015^{26}$ & 92.9 & 78.6 & NR \\
\hline Bahk et al. $2010^{27}$ & 100 & 100 & 4.3 \\
\hline Badge et al. $2009^{12}$ & 84.6 & 65.4 & NR \\
\hline Bradley et al. $2006^{18}$ & 89.0 & 67.0 & NR \\
\hline Bradley et al. $2013^{28}$ & 90.0 & 63.5 & NR \\
\hline Castagna et al. $2007^{29}$ & 100 & 100 & NR \\
\hline Eckenrode et al. $2009^{30}$ & 80.0 & 80.0 & NR \\
\hline Garret et al. $2017^{31}$ & 71.4 & NR & NR \\
\hline Hines et al. $2018^{37}$ & 87.5 & NR & NR \\
\hline Katthagen et al. $2017^{33}$ & 78.6 & 78.6 & NR \\
\hline Kercher et al. $2019^{35}$ & 93.8 & 62.5 & NR \\
\hline Kim et al. $2003^{36}$ & 100 & NR & NR \\
\hline Kraeutler et al. $2018^{34}$ & 68.2 & 50.0 & 8.6 \\
\hline Lacheta et al. $2019^{32}$ & 100 & 100 & NR \\
\hline Lenart et al. $2012^{19}$ & 100 & 100 & NR \\
\hline Mair et al. $1998^{9}$ & 100 & 100 & NR \\
\hline McClincy et al. $2015^{38}$ & 85.4 & 60.4 & NR \\
\hline McClincy et al. $2020^{39}$ & 89.0 & 76.8 & NR \\
\hline Papendick and Savoie $1995^{20}$ & 100 & NR & NR \\
\hline Radkowski et al. $2008^{40}$ & 88.8 & 73.5 & NR \\
\hline Robins et al. $2017^{41}$ & 91.9 & NR & NR \\
\hline Wanich et al. $2012^{42}$ & 91.7 & 91.7 & 5.9 \\
\hline Wolf and Eakin $1998^{43}$ & 100 & NR & NR \\
\hline Wooten et al. $2015^{44}$ & 80.0 & 68.0 & NR \\
\hline
\end{tabular}

Abbreviation: NR, not reported.

to the same level of play. In overhead athletes, the overall rate of return to play was $85.2 \%$ to $100 \%$, with $55.6 \%$ to $100 \%$ returning to the same level of play (Table 3).

\section{RTP Criteria}

Overall return-to-play criteria were reported in the majority of studies $(60 \%)$, with the most commonly report item being restoration of shoulder strength

Table 3. Rate of Return to Play in Collision and Overhead Athletes

\begin{tabular}{|c|c|c|c|}
\hline Reference & Return to Play (\%) & $\begin{array}{c}\text { Return to Play at Same or } \\
\text { Higher Level }(\%)\end{array}$ & Time $(\mathrm{mo})$ \\
\hline \multicolumn{4}{|l|}{ Collision athletes } \\
\hline Arner et al. $2015^{26}$ & 92.9 & 78.6 & NR \\
\hline Badge et al. $2009^{12}$ & 100 & 100 & 4.3 \\
\hline Bradley et al. $2006^{18}$ & 86.3 & 74.5 & NR \\
\hline Bradley et al. $2013^{28}$ & 90.6 & 69.2 & NR \\
\hline Castagna et al. $2007^{29}$ & 100 & 100 & $\mathrm{NR}$ \\
\hline Eckenrode et al. $2009^{30}$ & 80.0 & 80.0 & NR \\
\hline Kim et al. $2003^{36}$ & 100 & NR & NR \\
\hline Lacheta et al. $2019^{32}$ & 100 & 100 & NR \\
\hline Lenart et al. $2012^{19}$ & 100 & 100 & NR \\
\hline Mair et al. $1998^{9}$ & 100 & 100 & NR \\
\hline Robins et al. $2017^{41}$ & 91.9 & NR & NR \\
\hline Wolf and Eakin $1998^{43}$ & 100 & NR & NR \\
\hline \multicolumn{4}{|l|}{ Overhead athletes } \\
\hline Kercher et al. $2019^{35}$ & 93.8 & 62.5 & NR \\
\hline Kim et al. $2003^{36}$ & 100 & $\mathrm{NR}$ & NR \\
\hline Lenart et al. $2012^{19}$ & 100 & 100 & NR \\
\hline McClincy et al. $2015^{38}$ & 85.4 & 60.4 & NR \\
\hline Papendick and Savoie $1995^{20}$ & 100 & NR & NR \\
\hline Radkowski et al. $2008^{40}$ & 85.2 & 55.6 & NR \\
\hline Wanich et al. $2012^{42}$ & 91.7 & 91.7 & 5.9 \\
\hline Wolf and Eakin $1998^{43}$ & 100 & NR & NR \\
\hline
\end{tabular}

Abbreviation: NR, not reported. 
Table 4. Return-to-Play Criteria

\begin{tabular}{lr}
\hline Overall & $15(60)$ \\
Strength & $11(44)$ \\
Range of motion & $10(40)$ \\
Time & $9(36)$ \\
Pain & $5(20)$ \\
Sport-specific rehabilitation & $4(16)$ \\
Proprioception & $1(5)$ \\
\hline Data are $\mathrm{n}(\%)$. &
\end{tabular}

$(44 \%)$. There was a small discrepancy in reported time of return, with 9 studies $(36 \%)$ reporting return at either 4 or 6 months, 6 months being the most commonly used time point $(24 \%)$. Other criteria including range of motion (ROM) $(40 \%)$, absence of pain $(20 \%)$, completion of sport-specific rehabilitation $(16 \%)$, and proprioception $(4 \%)$ were also reported. The mean RTP quality of evidence was 1.8 (range 0 to 4) (Table 4).

\section{Discussion}

The most important finding from this systematic review of the literature was a high rate of return to sport after arthroscopic posterior shoulder stabilization, with the majority of patients returning to the same level of play. Both collision and overhead athletes returned to play at high rates, but one third of overhead athletes were unable to return to their preinjury status. However, there is inadequate reporting of RTP criteria in the current literature. Additionally, it remains unclear in the literature when it is safe to return to play, with appreciable variations in when athletes could return. Time, strength, and ROM were the most commonly reported criteria for RTP, but a few studies used sportsspecific rehabilitation protocols.

Return to play is a key and desirable outcome after orthopaedic procedures. Healthy, active patients place great importance on returning to sports and returning to the same (or higher) level as preinjury. ${ }^{24}$ This systematic review demonstrated that RTP was achievable at a high rate after posterior shoulder stabilization. ${ }^{9,18,19,25-44}$ Bradley et al. ${ }^{28}$ evaluated 200 shoulders, diagnosed with unidirectional recurrent posterior shoulder instability, and noted that $90 \%$ returned to play. Both McClincy et al. ${ }^{38}$ and Radkowski et al. ${ }^{40}$ compared surgical outcomes and RTP rates in throwing and nonthrowing athletes. McClincy et al. ${ }^{38}$ reported that $86 \%$ of the athletes were able to return to play, with no significant difference between the throwing and nonthrowing athletes. Radkowski et al. ${ }^{40}$ reported that throwing and nonthrowing athletes returned to play at $85 \%$ and $91 \%$, respectively. These reported findings indicate that athletes of varying sports-specific motions return to play at high levels after posterior shoulder stabilization. However, it is still unknown whether these players were able to sustain their activity level, or what the impact of returning to play had on the durability of their posterior shoulder stabilization. It is worth mentioning that the reported rates compare well to other commonly performed sports surgeries such as anterior shoulder stabilization, anterior cruciate ligament reconstruction, and medial patellofemoral ligament surgery. ${ }^{23,45-47}$

Although several studies reported high rates of RTP, the number of athletes who return to the same or higher level varies. Studies by Bradley et al. ${ }^{18}$ and Bahk et al. ${ }^{27}$ reported similar RTP rates. However, return to the same or higher level rates was $\sim 20 \%$ lower, suggesting excellent results after surgery but room for improvement, and future research with regard to returning with the same ability before the athlete's injury. ${ }^{18,27}$ A study in $2009^{30}$ documented the outcomes and RTP rates for 5 collegiate Division I wrestlers after posterior shoulder stabilization. Four of the 5 athletes returned to their preinjury status (the fifth graduated from college), including 2 achieving AllAmerican honors. ${ }^{30}$ Although they make up a small sample size, these wrestlers were able to recover fully and perform at an elite level. Lacheta et al. ${ }^{32}$ followed athletes after arthroscopic posterior bony Bankart bridge repair technique and found all 9 athletes returned to play and achieved their previous athletic level. The type of sport had no significance on the outcomes or the ability to return to the same or higher level of performance among this cohort.

Several studies reported data on overhead and collision subcohorts, determining whether the physical motion of the shoulder or the type of sport had an impact on RTP rates and return to preinjury status. ${ }^{9} 18,19,26,28,38,40,42$ Our systematic review revealed that overhead and collision athletes returned to play at similar rates, but collision athletes were more likely than overhead athletes to return to the same or higher level. A study in 2015 assessed 56 American football players, concluding that $93 \%$ of the players successfully returned to sport. However, only $79 \%$ were capable of returning to their preinjury status. ${ }^{26}$ Another study in 2015 compared nonthrowing athletes to overhead throwing athletes, documenting similar RTP rates. Although overhead throwing athletes returned to play at a rate of $85 \%$, only $60 \%$ were able to return to the same or higher level. ${ }^{38}$ The discrepancy in return to preinjury status between collision and overhead athletes suggests the need for validated RTP criteria.

Most of the selected studies reported general criteria for RTP, with most reporting time, ROM, and strength as important components. The majority of the studies allowed RTP at 6 months. Developing a verified RTP guideline may potentially help decrease the rate of recurrence of instability. Several studies reported the rate of recurrence within each respective cohort after posterior shoulder stabilization. Bahk et al. ${ }^{27}$ and Hines 
et al. $^{37}$ reported $3.4 \%$ and $6 \%$ recurrence rates, respectively. Bradley et al. ${ }^{28}$ prospectively reviewed 200 patients, and 14 of the 200 required arthroscopic revision. A systematic review conducted in 2018 defined RTP criteria after operative stabilization for traumatic anterior shoulder instability in hopes of reducing the recurrence rate. ${ }^{48}$ Much like the Hurley et al. ${ }^{49}$ systematic review that reported RTP rates after anterior shoulder stabilization, creating a validated checklist for a safe RTP after posterior shoulder stabilization would help improve surgical outcomes, decrease recurrence rates, and help close the gap between RTP and rates of return to the same or higher level.

Several factors have been postulated and correlated with athletes who are unable to return to play, such as poor healing, timing of life events (graduation from high school or college), and loss of interest in the injury-causing sport. Bahk et al. ${ }^{27}$ evaluated clinical outcomes and attempted to identify predictors of success after arthroscopic posterior Bankart reconstruction, concluding that $96.6 \%$ of the cohort reported success, with $84.6 \%$ returning to sports. Of the 8 athletes who did not return to sports, $75 \%$ documented that it was because of their shoulder. ${ }^{27}$ A 2015 study evaluated American football players after arthroscopic stabilization of PSI, with a majority returning to sport. However, the study team reported that some players returned at a different level or not at all because of injuries other than PSI or because they chose to partake in a different sport. ${ }^{26}$ Military personal were evaluated for the presence of posterior glenoid bone loss after arthroscopic isolated stabilization of the posterior labrum, investigating its impact on return to duty, complications, and surgical outcomes. Patients were separated according to mean posterior glenoid bone loss: $<13.5 \%$, considered minimal loss, and $>13.5 \%$, considered subcritical. Patients with $>13.5 \%$ bone loss were less likely to return to full duty compared with those with minimal bone loss, $14.3 \%$ versus $8 \%$, respectively. ${ }^{37}$ Baseball players who underwent arthroscopic posterior labral repair were assessed for surgical outcomes and described a large RTP rate. However, pitchers were less likely than position players to return to preinjury levels $(41 \%$ versus $86 \%) .{ }^{35}$ This suggests that it may be harder to return to certain positions in baseball and potentially other major sports after PSI.

\section{Limitations}

Systematic reviews are vulnerable to limitations, including possible biases in the selected studies. Specific variables restrict the conclusions made from this study, including the retrospective composition, low level of evidence, and irregular reporting of RTP information. Because of limitations in the included studies' reporting, we were unable to analyze whether demographic factors or dominance of shoulder were potential risk factors for inability to RTP and return to the same or higher level. Additionally, we were unable to determine whether concomitant pathologies hindered an athlete's ability to return to play or return to their preinjury status. A few studies reported a mean followup $<12$ months, questioning the potential effect of a return at 4 months on overall outcomes and a return to athletic activities. Lastly, the long-term effects of loss of ROM and overconstraint could not be assessed given the short- and medium-term nature of the reviewed studies.

\section{Conclusion}

There is a high rate of return to sport after arthroscopic posterior shoulder stabilization, ranging from 4.3 to 8.6 months after surgery. Return to preinjury level is higher for collision athletes compared with overhead athletes. However, there is inadequate reporting on RTP criteria in the current literature, with no clear timeline for when it is safe to return to sport.

\section{References}

1. Mc LH. Posterior dislocation of the shoulder. J Bone Joint Surg Am 1952:24:584-590.

2. Boyd HB, Sisk TD. Recurrent posterior dislocation of the shoulder. J Bone Joint Surg Am 1972;54:779-786.

3. Antoniou J, Duckworth DT, Harryman DT 2nd. Capsulolabral augmentation for the the management of posteroinferior instability of the shoulder. J Bone Joint Surg Am 2000;82:1220-1230.

4. Provencher MT, LeClere LE, King S, et al. Posterior instability of the shoulder: Diagnosis and management. Am J Sports Med 2011;39:874-886.

5. Antosh IJ, Tokish JM, Owens BD. Posterior shoulder instability. Sports Health 2016;8:520-526.

6. Schwartz E, Warren RF, O'Brien SJ, Fronek J. Posterior shoulder instability. Orthop Clin North Am 1987;18: 409-419.

7. Fronek J, Warren RF, Bowen M. Posterior subluxation of the glenohumeral joint. J Bone Joint Surg Am 1989;71: 205-216.

8. Robinson CM, Seah M, Akhtar MA. The epidemiology, risk of recurrence, and functional outcome after an acute traumatic posterior dislocation of the shoulder. J Bone Joint Surg Am 2011;93:1605-1613.

9. Mair SD, Zarzour RH, Speer KP. Posterior labral injury in contact athletes. Am J Sports Med 1998;26:753-758.

10. Kaplan LD, Flanigan DC, Norwig J, Jost P, Bradley J. Prevalence and variance of shoulder injuries in elite collegiate football players. Am J Sports Med 2005;33: $1142-1146$.

11. DeLong JM, Jiang K, Bradley JP. Posterior instability of the shoulder: A systematic review and meta-analysis of clinical outcomes. Am J Sports Med 2015;43:1805-1817.

12. Badge R, Tambe A, Funk L. Arthroscopic isolated posterior labral repair in rugby players. Int J Shoulder Surg 2009;3:4-7. 
13. Tannenbaum E, Sekiya JK. Evaluation and management of posterior shoulder instability. Sports Health 2011;3: 253-263.

14. Burkhead WZ Jr, Rockwood CA Jr. Treatment of instability of the shoulder with an exercise program. J Bone Joint Surg Am 1992;74:890-896.

15. Hawkins RJ, Koppert G, Johnston G. Recurrent posterior instability (subluxation) of the shoulder. J Bone Joint Surg Am 1984;66:169-174.

16. Hovis WD, Dean MT, Mallon WJ, Hawkins RJ. Posterior instability of the shoulder with secondary impingement in elite golfers. Am J Sports Med 2002;30:886-890.

17. Bottoni CR, Franks BR, Moore JH, DeBerardino TM, Taylor DC, Arciero RA. Operative stabilization of posterior shoulder instability. Am J Sports Med 2005;33: 996-1002.

18. Bradley JP, Baker CL 3rd, Kline AJ, Armfield DR, Chhabra A. Arthroscopic capsulolabral reconstruction for posterior instability of the shoulder: A prospective study of 100 shoulders. Am J Sports Med 2006;34:1061-1071.

19. Lenart BA, Sherman SL, Mall NA, Gochanour E, Twigg SL, Nicholson GP. Arthroscopic repair for posterior shoulder instability. Arthroscopy 2012;28:1337-1343.

20. Papendick LW, Savoie FH 3rd. Anatomy-specific repair techniques for posterior shoulder instability. J South Orthop Assoc 1995:4:169-176.

21. OCEBM Levels of Evidence Working Group. The Oxford levels of evidence 2. Oxford Centre for Evidence-Based Medicine. Available at: https://www.cebm.ox.ac.uk/ resources/levels-of-evidence/ocebm-levels-of-evidenceO CEBM-Levels-of-Evidence-Working-Group.

22. Ramponi L, Yasui Y, Murawski CD, et al. Lesion size is a predictor of clinical outcomes after bone marrow stimulation for osteochondral lesions of the talus: A systematic review. Am J Sports Med 2017;45:1698-1705.

23. Zaman S, White A, Shi WJ, Freedman KB, Dodson CC. Return-to-play guidelines after medial patellofemoral ligament surgery for recurrent patellar instability: A systematic review. Am J Sports Med 2018;46:2530-2539.

24. Sonesson S, Kvist J, Ardern C, Österberg A, Silbernagel KG. Psychological factors are important to return to pre-injury sport activity after anterior cruciate ligament reconstruction: Expect and motivate to satisfy. Knee Surg Sports Traumatol Arthrosc 2017;25:1375-1384.

25. Andrieu K, Barth J, Saffarini M, et al. Outcomes of capsulolabral reconstruction for posterior shoulder instability. Orthop Traumatol Surg Res 2017;103:S189-S192.

26. Arner JW, McClincy MP, Bradley JP. Arthroscopic stabilization of posterior shoulder instability is successful in American football players. Arthroscopy 2015;31: 1466-1471.

27. Bahk MS, Karzel RP, Snyder SJ. Arthroscopic posterior stabilization and anterior capsular plication for recurrent posterior glenohumeral instability. Arthroscopy 2010;26: 1172-1180.

28. Bradley JP, McClincy MP, Arner JW, Tejwani SG. Arthroscopic capsulolabral reconstruction for posterior instability of the shoulder: A prospective study of 200 shoulders. Am J Sports Med 2013;41:2005-2014.

29. Castagna A, Snyder SJ, Conti M, Borroni M, Massazza G, Garofalo R. Posterior humeral avulsion of the glenohumeral ligament: A clinical review of 9 cases. Arthroscopy 2007;23:809-815.

30. Eckenrode BJ, Logerstedt DS, Sennett BJ. Rehabilitation and functional outcomes in collegiate wrestlers following a posterior shoulder stabilization procedure. J Orthop Sports Phys Ther 2009;39:550-559.

31. Garret J, Nourissat G, Hardy MB, et al. Painful posterior shoulder instability: Anticipating and preventing failure. A study in 25 patients. Orthop Traumatol Surg Res 2017;103: S199-S202.

32. Lacheta L, Goldenberg BT, Horan MP, Millett PJ. Posterior bony Bankart bridge technique results in reliable clinical 2 -year outcomes and high return to sports rate for the treatment of posterior bony Bankart lesions. Knee Surg Sports Traumatol Arthrosc 2019 [online ahead of print]. https://doi.org/10.1007/s00167-019-05783-X.

33. Katthagen JC, Tahal DS, Montgomery SR, Horan MP, Millett PJ. Association of traumatic and atraumatic posterior shoulder instability with glenoid retroversion and outcomes after arthroscopic capsulolabral repair. Arthroscopy 2017;33:284-290.

34. Kraeutler MJ, Aberle NS, Brown CC, Ptasinski JJ, McCarty EC. Clinical outcomes and return to sport after arthroscopic anterior, posterior, and combined shoulder stabilization. Orthop J Sports Med 2018;6: 2325967118763754.

35. Kercher JS, Runner RP, McCarthy TP, Duralde XA. Posterior labral repairs of the shoulder among baseball players: Results and outcomes with minimum 2-year follow-up. Am J Sports Med 2019;47:1687-1693.

36. Kim SH, Ha KI, Park JH, et al. Arthroscopic posterior labral repair and capsular shift for traumatic unidirectional recurrent posterior subluxation of the shoulder. J Bone Joint Surg Am 2003;85:1479-1487.

37. Hines A, Cook JB, Shaha JS, et al. Glenoid bone loss in posterior shoulder instability: Prevalence and outcomes in arthroscopic treatment. Am J Sports Med 2018;46: 1053-1057.

38. McClincy MP, Arner JW, Bradley JP. Posterior shoulder instability in throwing athletes: A case-matched comparison of throwers and non-throwers. Arthroscopy 2015;31: 1041-1051.

39. McClincy MP, Arner JW, Thurber L, Bradley JP. Arthroscopic capsulolabral reconstruction for posterior shoulder instability is successful in adolescent athletes. J Pediatr Orthop 2020;40:135-141.

40. Radkowski CA, Chhabra A, Baker CL 3rd, Tejwani SG, Bradley JP. Arthroscopic capsulolabral repair for posterior shoulder instability in throwing athletes compared with nonthrowing athletes. Am J Sports Med 2008;36:693-699.

41. Robins RJ, Daruwalla JH, Gamradt SC, et al. Return to play after shoulder instability surgery in National Collegiate Athletic Association Division I intercollegiate football athletes. Am J Sports Med 2017;45:2329-2335.

42. Wanich T, Dines J, Dines D, Gambardella RA, Yocum LA. 'Batter's shoulder': Can athletes return to play at the same level after operative treatment? Clin Orthop Relat Res 2012;470:1565-1570.

43. Wolf EM, Eakin CL. Arthroscopic capsular plication for posterior shoulder instability. Arthroscopy 1998;14: 153-163. 
44. Wooten CJ, Krych AJ, Schleck CD, Hudgens JL, May JH, Dahm DL. Arthroscopic capsulolabral reconstruction for posterior shoulder instability in patients 18 years old or younger. J Pediatr Orthop 2015;35:462-466.

45. Fabricant PD, Chin CS, Conte S, Coleman SH, Pearle AD, Dines JS. Return to play after anterior cruciate ligament reconstruction in major league baseball athletes. Arthroscopy 2015;31:896-900.

46. Memon M, Kay J, Cadet ER, Shahsavar S, Simunovic N, Ayeni OR. Return to sport following arthroscopic Bankart repair: A systematic review. I Shoulder Elbow Surg 2018;27:1342-1347.
47. Ialenti MN, Mulvihill JD, Feinstein M, Zhang AL, Feeley BT. Return to play following shoulder stabilization: A systematic review and meta-analysis. Orthop J Sports Med 2017;5:2325967117726055.

48. Ciccotti MC, Syed U, Hoffman R, Abboud JA, Ciccotti MG, Freedman KB. Return to play criteria following surgical stabilization for traumatic anterior shoulder instability: A systematic review. Arthroscopy 2018;34:903-913.

49. Hurley ET, Montgomery C, Jamal MS, et al. Return to play after the Latarjet procedure for anterior shoulder instability: A systematic review. Am J Sports Med 2019;47: 3002-3008. 